\title{
EchoGéo
}

6 | 2008

La Guyane

\section{Le risque cyclonique dans l'Atlantique en 2008}

Analyse des ouragans Gustav à Ike et comparaison avec Katrina

\section{Sandra Rome}

\section{(2) OpenEdition}

Journals

Édition électronique

URL : https://journals.openedition.org/echogeo/7613

DOI : $10.4000 /$ echogeo.7613

ISSN : 1963-1197

Éditeur

Pôle de recherche pour l'organisation et la diffusion de l'information géographique (CNRS UMR 8586)

Référence électronique

Sandra Rome, "Le risque cyclonique dans I'Atlantique en 2008 », EchoGéo [En ligne], 6 | 2008, mis en ligne le 06 octobre 2008, consulté le 31 juillet 2021. URL : http://journals.openedition.org/echogeo/ 7613 ; DOI : https://doi.org/10.4000/echogeo.7613

Ce document a été généré automatiquement le 31 juillet 2021.

EchoGéo est mis à disposition selon les termes de la licence Creative Commons Attribution - Pas d'Utilisation Commerciale - Pas de Modification 4.0 International (CC BY-NC-ND) 


\section{Le risque cyclonique dans l'Atlantique en 2008}

Analyse des ouragans Gustav à Ike et comparaison avec Katrina

\section{Sandra Rome}

\section{Introduction}

1 Septième cyclone nommé de la saison de l'Atlantique, Gustav, ouragan de catégorie 1 sur l'échelle de Saffir-Simpson, est présenté par les média comme le successeur de Katrina qui a sévèrement endeuillé et endommagé une partie de la ville de la Nouvelle Orléans et des côtes de Louisiane en 2005. Gustav frappe le sud-ouest de la République de Haïti le mardi 26 août 2008, avec des vents atteignant $145 \mathrm{~km} / \mathrm{h}$. Le cyclone s'affaibli en tempête tropicale après avoir traversé la péninsule ouest d'Hispaniola, puis reprend de la vigueur en passant au stade d'ouragan de catégorie 2 puis 3 en direction de la Jamaïque et de Cuba, avant de se diriger vers le Golfe du Mexique et la Louisiane. Finalement Gustav provoque beaucoup moins de dégâts que prévu aux Etats-Unis, en grande partie parce que l'ouragan s'est affaiblit, mais aussi grâce aux mesures de prévention et d'évacuation du littoral américain. Mais d'autres ouragans se déchaînent sur Haïti, sévèrement endeuillé successivement par les cyclones Gustav, Hanna et Ike fin août, début septembre 2008.

2 L'objet de ce travail est d'analyser d'une part, l'aléa cyclonique de différents systèmes météorologiques remarquables qui affectent l'arc des Antilles et le Golfe du Mexique lors de Katrina en 2005 et surtout en 2008 ; d'autre part, la vulnérabilité liée aux espaces affectés est rapidement abordée avant de comparer le risque cyclonique.

Ce dossier «sur le vif» fait d'abord le point sur les cyclones de l'Atlantique, avant de présenter les données et les méthodes utilisées, puis la chronique météorologique des ouragans de 2008 et enfin, de proposer une analyse du risque dans certains états de l'archipel caribéen et aux Etats-Unis. 


\section{Analyse des cyclones de l'océan Atlantique : données et méthode}

\subsection{Une terminologie internationale}

4 La terminologie internationale désigne par cyclone, une « perturbation atmosphérique tourbillonnaire, de grande échelle, due à une chute importante de la pression atmosphérique ».

5 Ces perturbations météorologiques se rencontrent dans les régions tropicales et sont caractérisées par des pluies abondantes; les vents très violents, atteignant $350 \mathrm{~km} / \mathrm{h}$. autour de l'oeil, tournent dans le sens horaire dans l'hémisphère sud et le sens antihoraire dans l'hémisphère nord. Dans l'Atlantique nord, on emploie les termes de dépression tropicale, tempête tropicale et ouragan en fonction de la vitesse maximale des vents (tab. 1), les trois stades correspondant au terme générique de cyclone.

Tableau 1 - Les caractéristiques aérologiques des cyclones tropicaux dans l'Atlantique nord et les catégories d'ouragan selon l'échelle de Saffir et Simpson.

\begin{tabular}{|c|c|c|c|c|c|c|c|}
\hline $\begin{array}{l}\text { Stades du } \\
\text { cyclone }\end{array}$ & Dépression & $\begin{array}{l}\text { Tempête } \\
\text { tropicale }\end{array}$ & $\begin{array}{c}\text { Ouragan } \\
\text { catégorie } \\
1\end{array}$ & $\begin{array}{c}\text { Ouragan } \\
\text { catégorie } \\
2\end{array}$ & $\begin{array}{c}\text { Ouragan } \\
\text { catégorie } \\
3\end{array}$ & $\begin{array}{c}\text { Ouragan } \\
\text { catégorie } \\
\mathbf{4}\end{array}$ & $\begin{array}{c}\text { Ouragan } \\
\text { catégorie } \\
5\end{array}$ \\
\hline Vent $(\mathrm{km} / \mathrm{h})$ & $\leq 63$ & $63-118$ & $118-152$ & $152-178$ & $178-210$ & $210-250$ & $\geq 250$ \\
\hline Vent (nœuds) & $\leq 34$ & $34-63$ & $63-82$ & $83-95$ & $96-112$ & $113-134$ & $\geq 135$ \\
\hline Vent (Beaufort) & force 7 & force 8-11 & force 12 & force 12 & force 12 & force 12 & force 12 \\
\hline $\begin{array}{l}\text { Identification } \\
\text { Stade } \\
N^{\circ} \text { ou prénom } \\
\text { (année) }\end{array}$ & $\begin{array}{c}\text { Numéro: } \\
\text { Dépression } \\
n^{\circ} 1\end{array}$ & $\begin{array}{l}\text { Prénom : } \\
\text { Tempête } \\
\text { "Hugo" }\end{array}$ & $\begin{array}{c}\text { Prénom : } \\
\text { Ouragan } \\
\text { "Lili" } \\
(2002)\end{array}$ & $\begin{array}{c}\text { Prénom : } \\
\text { Ouragan } \\
\text { "Frances" } \\
(2004)\end{array}$ & $\begin{array}{c}\text { Prénom : } \\
\text { Ouragan } \\
\text { "Jeanne" } \\
(2004)\end{array}$ & $\begin{array}{l}\text { Prénom : } \\
\text { Ouragan } \\
\text { "Dennis" } \\
(2005)\end{array}$ & $\begin{array}{c}\text { Prénom : } \\
\text { Ouragan } \\
\text { "Katrina" } \\
(2005)\end{array}$ \\
\hline Pression $(\mathrm{hPa})$ & & & $>980$ & $965-979$ & $945-964$ & $920-944$ & $<920$ \\
\hline $\begin{array}{l}\text { Elévation du } \\
\text { niveau de la } \\
\text { mer }(m)\end{array}$ & & & 1 à 1,7 & 1,8 à 2,6 & 2,7 à 3,8 & 3,9 à 5,6 & $>$ à 5,6 \\
\hline
\end{tabular}

6 Les ouragans sont subdivisés en cinq catégories, selon une échelle formulée en 1971 par Herbert Saffir et Robert Simpson. Cette échelle dite Saffir-Simpson, est une évaluation fondée sur l'intensité des vents et des dégâts potentiels des ouragans. Pour davantage de détails sur l'échelle Saffir-Simpson, se reporter au National Hurricane Center, sis à Miami, à l'adresse : http://www.nhc.noaa.gov/aboutsshs.shtml

\subsection{Données et méthode d'analyse du risque cyclonique}

7 Les données sur l'intensité des vents (nœuds convertis en $\mathrm{km} / \mathrm{h}$ ) et la pression atmosphérique minimale (en $\mathrm{hPa}$ ) des cyclones proviennent du Centre National américain des ouragans (National Hurricane Center) de l'Administration Américaine de l'Atmosphère et l'Océan (National Oceanic and Atmospheric Administration). Elles sont rendues accessibles de deux manières : 1/ par Thomas R. Metcalf de l'Université de Hawaï qui propose également la trajectoire des cyclones (http:// www.solar.ifa.hawaii.edu/Tropical/index.wml) et 2/via le site Weather Underground (http://www.wunderground.com/hurricane/at2008.asp). Les trajectoires de cyclones 
montrent également l'intensité spatio-temporelle des événements. Deux années sont particulièrement étudiées, les saisons 2008 (juin à septembre) et 2005 (juin à octobre).

8 L'aléa cyclonique est étudié en comparant l'intensité des vents et la pression atmosphérique minimale mesurée au centre (œil) de plusieurs épisodes cycloniques, du stade de dépression tropicale (Jour 1 à $9 \mathrm{~h}$ GMT) à la fin de l'épisode, c'est-à-dire lorsque que le système s'affaiblit au stade de dépression et sort du domaine tropical. Le premier jour (J1) présente un décalage entre événements; ceci est dû au passage de la dépression tropicale au stade de tempête, contrairement au Jour 2. Généralement les mesures de vent sont effectuées quatre fois par jour (3, 9, 15, 21h GMT), et plus si le cyclone se renforce brutalement entre deux mesures ( 6 ou $18 \mathrm{~h}$ GMT).

La vulnérabilité des espaces est évaluée en tenant compte du nombre de décès et, quand cela est possible, d'une estimation des coûts occasionnés. La prise en compte des mesures de prévention et d'évacuation préalable n'est pas exploitée ici.

10 La saison cyclonique 2008 de l'océan Atlantique est relativement peu active, avec trois ouragans dépassant la catégorie 2.

\section{Une saison cyclonique 2008 intense dans l'Atlantique}

\subsection{Evolution spatio-temporelle de Gustav ( 25 août-2 septembre 2005)}

11 Le cyclone Gustav est issu d'une zone dépressionnaire qui se développe sur l'Atlantique nord, du 20 au 22 août, assez loin à l'est des Petites Antilles, mais restant peu organisée. Après être passée sur la moitié sud de l'arc antillais les 23 et 24 , occasionnant près de $100 \mathrm{~mm}$ de pluies et de nombreux orages sur Sainte-Lucie et la Martinique, elle est classée en matinée du 25 dépression tropicale au sud de la République Dominicaine, puis tempête tropicale dès que des mesures le confirmant sont rapportées par l'avion de reconnaissance (Météo France, 2008).

12 Le cyclone atteint l'intensité d'ouragan par $17,20^{\circ} \mathrm{N}$ et $71,90^{\circ} \mathrm{W}$ en cours de nuit du 25 au 26 août, à une centaine de kilomètres au sud de la république de Haïti vers laquelle il se dirige. La pression la plus faible enregistrée est alors de $984 \mathrm{hPa}$ et le vent de 85 nœuds $(157 \mathrm{~km} / \mathrm{h})$. L'ouragan Gustav traverse le sud-ouest de Haïti et s'affaiblit au stade de tempête tropicale dans la nuit du 26 au 27 août, avec des vents de 70 nœuds $(130 \mathrm{~km} / \mathrm{h})$ et une pression de $994 \mathrm{hPa}$.

13 Gustav se dirige ensuite vers la Jamaïque qu'il atteint et traverse le 28 , avec des vents d'environ 70 nœuds et une pression près de l'œil passant de 999 à $983 \mathrm{hPa}$.

Le 29 vers $21 \mathrm{~h}\left(19.20^{\circ} \mathrm{N}, 79.70^{\circ} \mathrm{W}\right)$ Gustav évolue de nouveau en ouragan de catégorie 1 (vents de 75 nœuds, pression de $980 \mathrm{hPa}$ ), frappe les îles Cayman, se renforce en quelques heures en ouragan de classe 4 sur l'échelle Saffir-Simpson, avec des vents maximum soutenus de 150 nœuds $(277 \mathrm{~km} / \mathrm{h})$ et une pression qui se creuse à $942 \mathrm{hPa}$ en début de nuit du 30 août ( $21 \mathrm{~h} \mathrm{GMT}$ ), par $22,10^{\circ}$ de latitude nord et $82,90^{\circ} \mathrm{W}$; il traverse l'île de la Jeunesse et l'ouest de Cuba, à proximité de La Havane.

15 Affaibli par son passage sur la terre cubaine, Gustav rejoint le Golfe du Mexique et se dirige vers la Louisiane qu'il atteint le $1^{\text {er }}$ septembre, après avoir faibli en ouragan de 
classe 2 dans la région de Lafayette, un peu à l'ouest de la Nouvelle Orléans. Il perd ensuite ses caractéristiques de cyclone tropical dans l'intérieur des terres de l'extrême est du Texas et sur la Louisiane, où il provoque de fortes pluies, ainsi que sur les états du Mississippi, Arkansas et Missouri.

Gustav suit une trajectoire dans les eaux chaudes de la mer des Antilles et du Golfe du Mexique (fig. 1).

Figure 1 - Trajectoire et intensité des vents du cyclone Gustav du 25 août au 2 septembre 2008

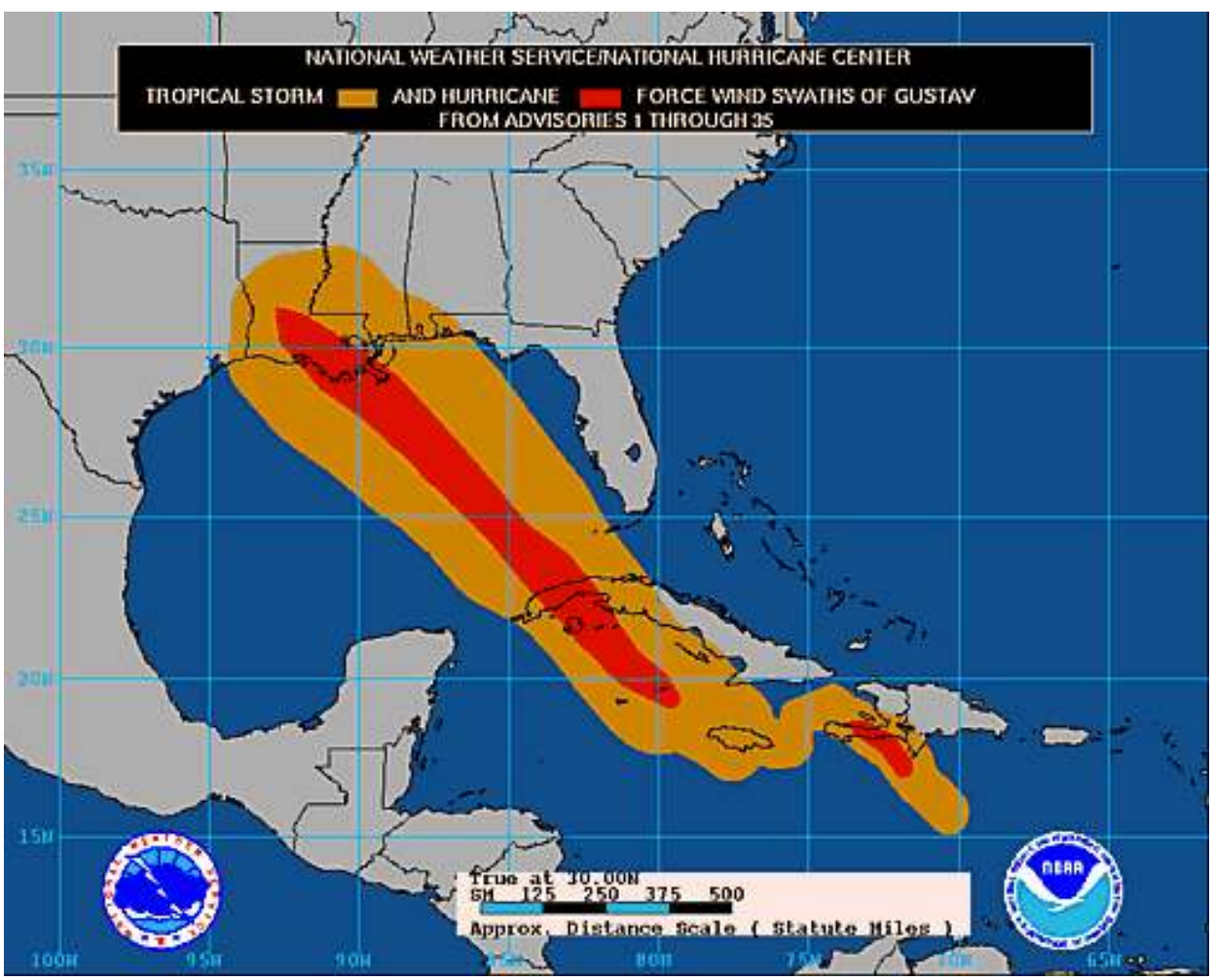

Source : NOAA/NHC, 2008 - Pour voir l'animation, suivre le lien : http://www.nhc.noaa.gov/archive/ 2008/graphics/al07/loop_S.shtml

17 Le cyclone Gustav a entraîné la mort de 8 personnes en République Dominicaine, 66 à Haïti, 11 en Jamaïque et 7 en Louisiane, soit 92 au total. L'anticipation des mesures de prévention et les nombreuses évacuations organisées dans cet état américain, ont permis de réduire le nombre potentiel de victimes, leçon tirée du passage dévastateur de Katrina tout juste trois ans plus tôt.

\subsection{L'ouragan Hanna affecte Haïti et la côte atlantique des États- Unis}

Du 28 août au 7 septembre 2008, la zone dépressionnaire qui se développe sur l'Atlantique au large nord-est des Petites Antilles les 26 et 27 août, évolue en dépression tropicale dans la nuit du 27 au 28 (positionnée par $19,80^{\circ} \mathrm{N}$ et $57,90^{\circ} \mathrm{W}$ ), puis vite tempête (pression de $1002 \mathrm{hPa}$, vitesse du vent de $68 \mathrm{~km} / \mathrm{h}$ ) et se dirige vers le nordouest à faible vitesse ; elle passe à $300 \mathrm{~km}$ environ au nord-est du nord de l'arc antillais, accompagnée de nombreuses pluies orageuses. Assez peu organisée jusqu'au 31, la tempête tropicale $\underline{\text { Hanna }}$ aborde l'archipel des Bahamas avec une pression qui se creuse 
à $999 \mathrm{hPa}$ et des vents de $111 \mathrm{~km} / \mathrm{h}$. Elle s'intensifie alors le $1^{\mathrm{er}}$ septembre et devient temporairement ouragan; le vent maximal soutenu atteint $148 \mathrm{~km} / \mathrm{h}$, la pression minimale est de $978 \mathrm{hPa}$ vers $3 \mathrm{~h}$ GMT le 2 septembre. Hanna effectue une boucle au sud des Bahamas et à proximité du nord de Haïti, une fois redevenue forte tempête. Puis elle traverse tout l'archipel des Bahamas dans sa remontée vers le nord-ouest avant d'arriver sur les états américains de Caroline du Nord et de Virginie dans la nuit du 5 au 6. Après être passée sur Washington et New York, elle perd ses caractéristiques tropicales le 7 au matin sur les états de la Nouvelle Angleterre (Météo France, 2008).

Suite aux inondations engendrées par les fortes précipitations, Hanna tue environ 500 personnes dans le nord de Haïti, petit pays déjà touché par Gustav six jours plus tôt. L'animation satellitale (fig. 3) montre l'évolution spatio-temporelle des deux cyclones de part et d'autre de l'île d'Hispaniola.

Figure 2 - Animation satellite des ouragans Gustav et Hanna sur l'Atlantique nord et le Golfe du Mexique

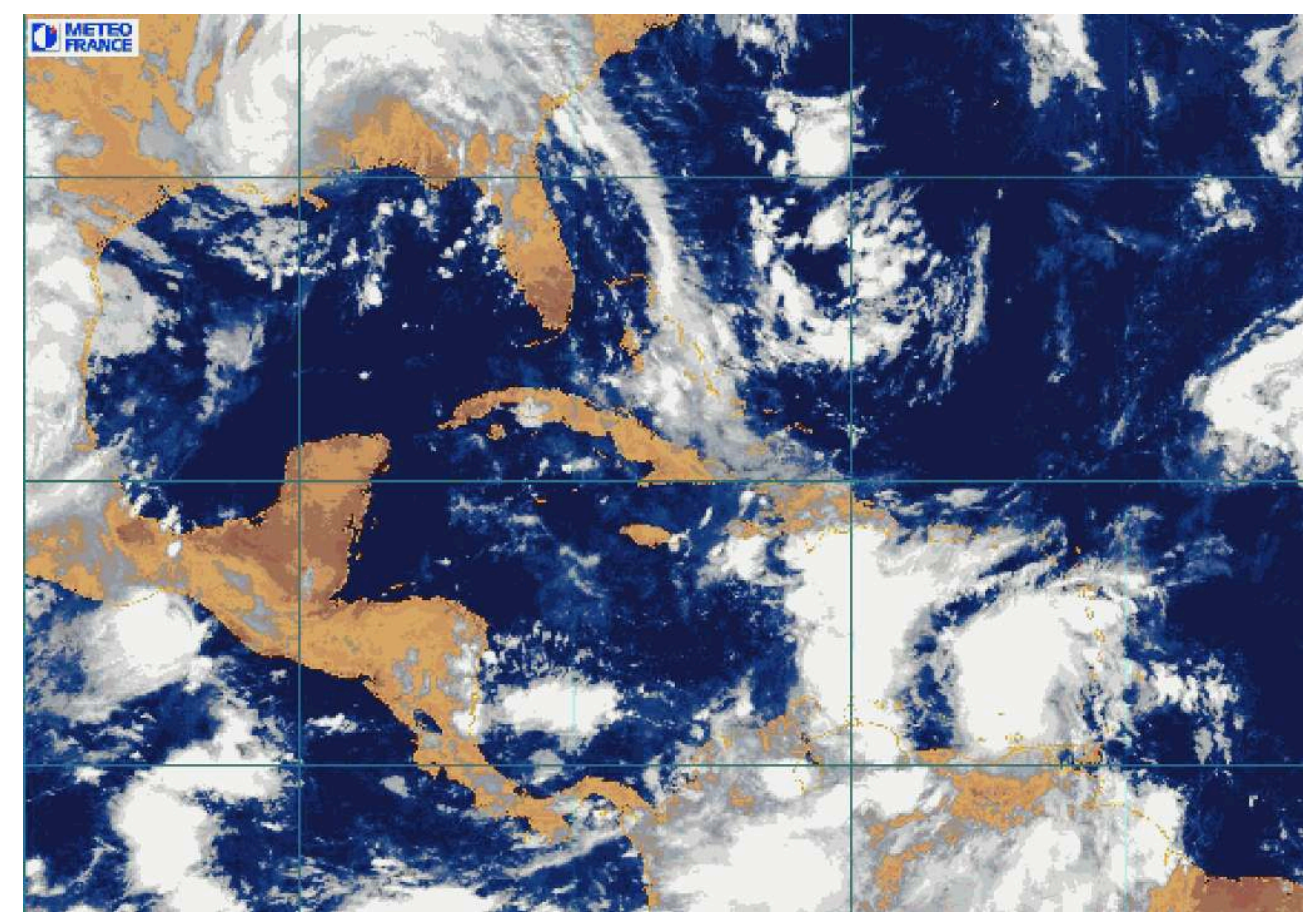

Source Météo France, 2008 - Pour voir l'animation sur la trajectoire des cyclones Gustav et Hanna http://www.meteo.fr/temps/domtom/antilles/pack-public/cyclone/Gustav_Hanna.gif

21 Les effets de Hanna sont passés quasiment inaperçus car occultés par le gigantisme de Ike qui la succède ; et pourtant, Hanna a tué 500 personnes à Haïti, tandis que les media restent focalisés sur les potentiels importants dégâts à venir de Ike sur les installations pétrolières texanes !

\subsection{L'ouragan Ike, le plus long de la saison, atteint la catégorie 4}

La perturbation qui naît entre l'archipel du Cap Vert et les Petites Antilles devient vite la tempête tropicale $\underline{I k e}$ à un peu plus de $2000 \mathrm{~km}$ au large de l'arc antillais. Ouragan dès le 3 septembre 2008 à $1000 \mathrm{~km}$ au nord-est de l'archipel antillais, il s'intensifie de manière explosive et atteint la catégorie 4 dans la nuit du 3 au 4 . La pression minimale 
n'est que de $935 \mathrm{hPa}$ et le vent maximal soutenu de $230 \mathrm{~km} / \mathrm{h}$ (125 noeuds). Ike poursuit sa route vers l'ouest, mais s'affaiblit en ouragan 3 lorsqu'il passe à $500 \mathrm{~km}$ au nord de Saint-Martin le 5 (fig. 3). Une inflexion vers le sud-ouest, comme Hanna quelques jours plus tôt, le fait se diriger vers le sud de l'archipel des Bahamas et le nord de Haïti déjà durement éprouvé: quatre cyclones consécutifs touchant le même territoire, c'est quasiment de l'inédit. L'ouragan de nouveau de classe 4 touche sévèrement l'île de Cuba, qu'il traverse de part en part du 7 au 9 septembre. Affaibli par son passage sur les terres, c'est un ouragan de classe 1 qui débouche dans le Golfe du Mexique, mettant le cap sur le Texas, qu'il atteint dans la nuit du 12 au 13 avec l'intensité de catégorie 2. Ike frappe durement Galveston (5 morts) et la région de Houston, avant de pénétrer dans les terres et de perdre son statut de cyclone tropical sur l'Arkansas, le Missouri, l'Indiana et l'Illinois (Météo France, 2008 ; AFP, 14/09/2008).

Figure 3 - Trajectoire et intensité des vents de l'ouragan lke du $1^{\mathrm{er}}$ au 14 septembre 2008

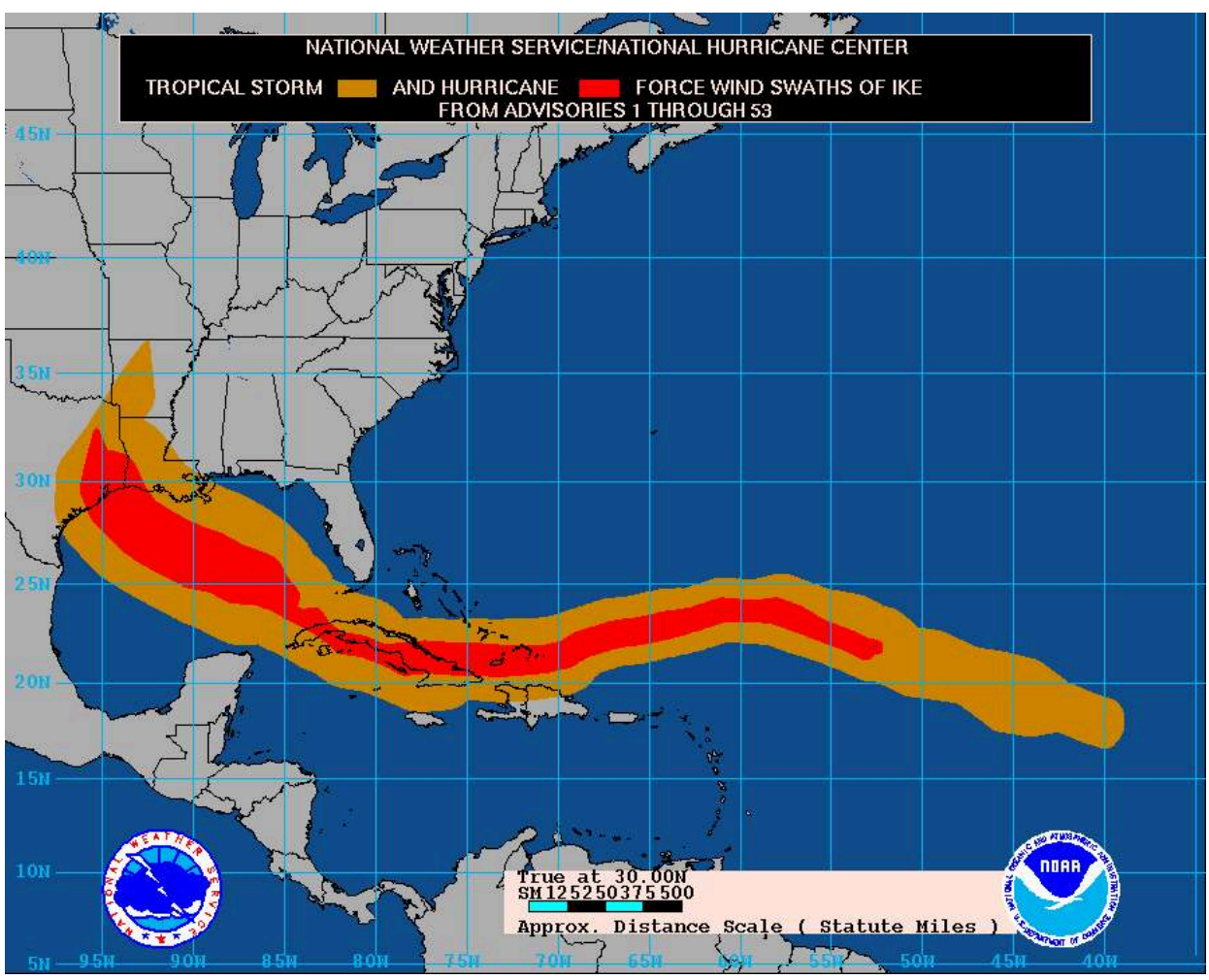

Source : NOAA/NCEP/NHC, 2008 - Pour voir l'animation suivre le lien : http://www.nhc.noaa.gov/ archive/2008/graphics/al09/loop_S.shtml

Ike présente une grande longévité, 14 jours. Il a tué, directement ou indirectement (chutes d'arbres, électrocution) 66 personnes à Haïti, 4 à Cuba, 52 aux Etats-Unis (AFP, 14/09/2008).

Ike est suivi d'une perturbation africaine, classée dépression tropicale (DT 10) juste au sud des îles du Cap Vert le 2 septembre, qui devient très vite une tempête tropicale baptisée Joséphine, et se déplace vers l'ouest. Le vent maximal soutenu atteint $100 \mathrm{~km} / \mathrm{h}$ (55 noeuds) le 3 la pression minimale est de $994 \mathrm{hPa}$. Fort heureusement, l'environnement atmosphérique hostile l'affaiblit les 4 et 5 septembre, et la déstructure le 6 au matin, à plus de $2500 \mathrm{~km}$ des Antilles. 


\subsection{L'augmentation de l'intensité des cyclones est en partie liée au réchauffement des surfaces océaniques}

La saison cyclonique 2008 peut être considérée comme assez active dans l'Atlantique nord, avec la présence de deux ouragans de catégorie 3 et plus (fig. 4), mais leur nombre est inférieur à la moyenne annuelle 1950-2000 (13 cyclones baptisés) pour l'océan Atlantique.

Figure 4 - Trajectoire des cyclones de la saison 2008 dans l'atlantique nord et le Golfe du Mexique

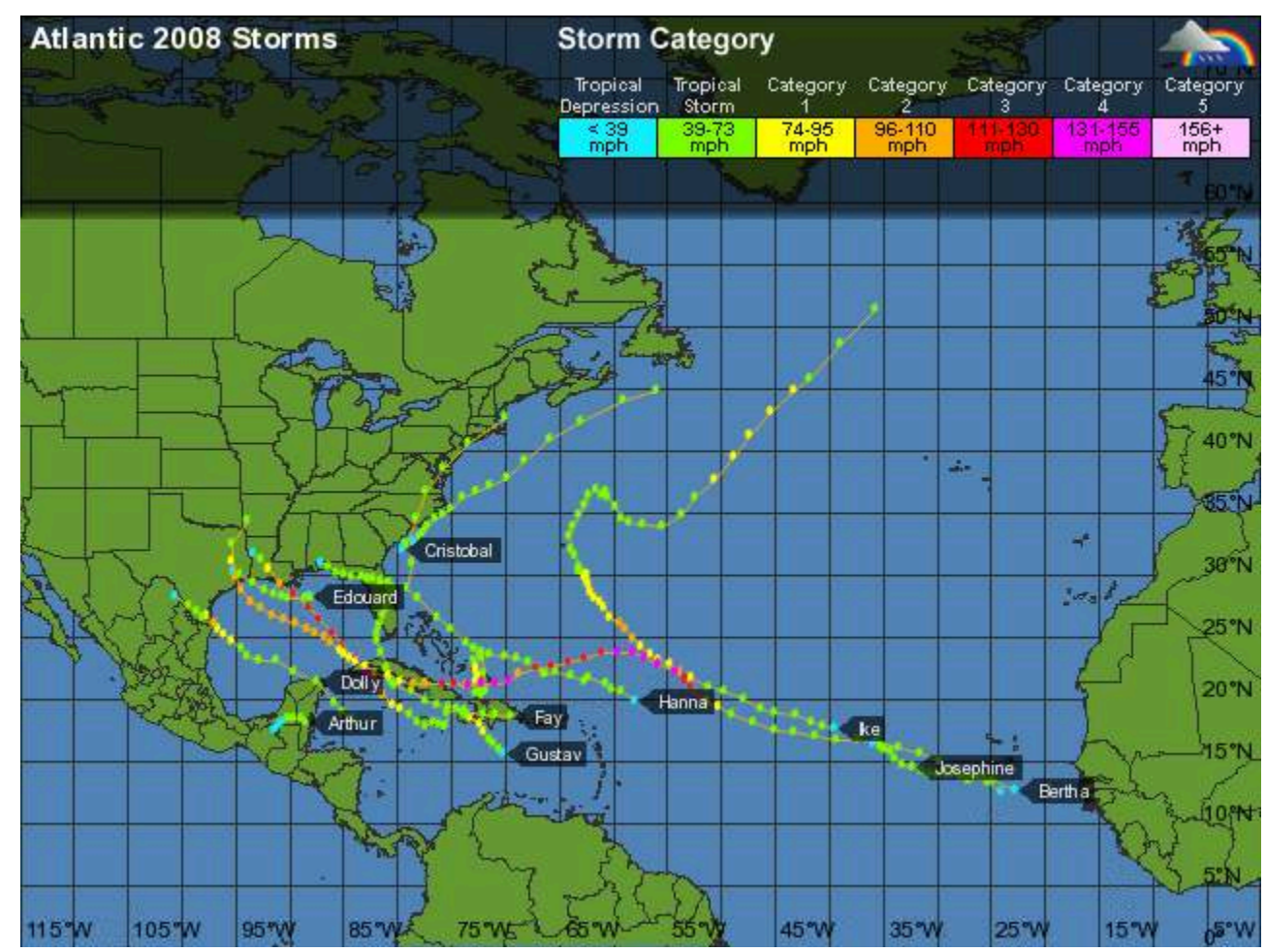

Source : Weather Underground, 2008 - Pour visionner la carte, suivre le lien : http:// www.wunderground.com/hurricane/at2008.asp?MR=1

Pour observer un cyclone en particulier, cliquer ensuite sur son nom et la trajectoire apparaît.

En comparaison, la saison cyclonique 2005 a battu tous les records avec 28 tempêtes tropicales (ancien record de 21 en 1933), 15 ouragans (12 en 1969) dont 4 de catégorie 5 (Emily, Katrina, Rita et Wilma) (fig. 5). 
Figure 5 - Trajectoire des cyclones de la saison 2005 dans l'atlantique nord et le Golfe du Mexique

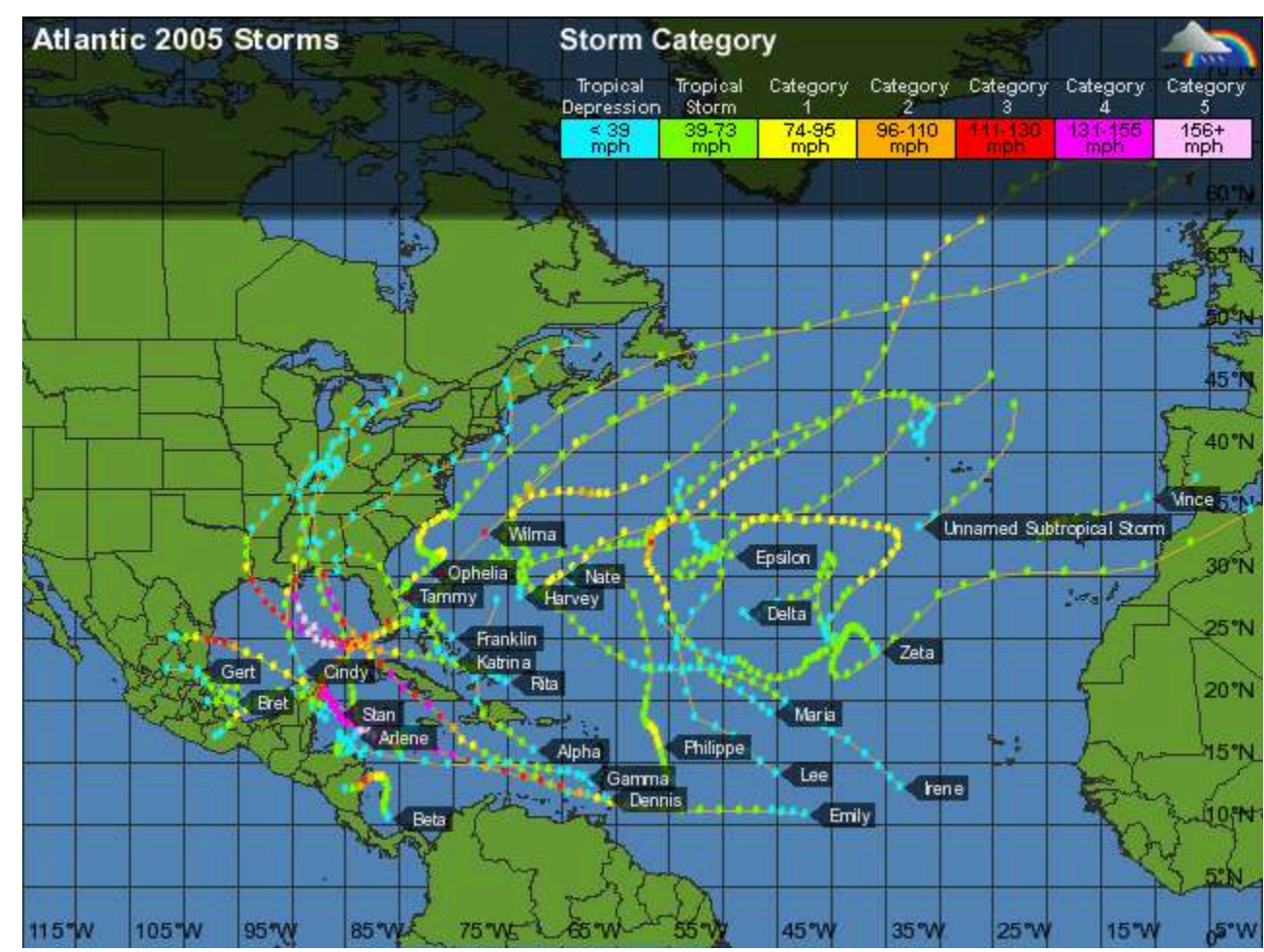

Source : Weather Underground, 2008. Pour visionner la carte, suivre le lien : http://www.wunderground.com/hurricane/at2005.asp?MR=1

1995 (Webster et al. 2005 ; Holland \& Webster, 2007), les causes sous-jacentes de cette augmentation restent incertaines (Saunders \& Lea, 2008). Plusieurs études récentes sur les cyclones tropicaux de l'Atlantique nord montrent une corrélation entre l'augmentation de l'activité cyclonique et le réchauffement de la Température de Surface Océanique. Emanuel (2005) montre qu'une augmentation de $0,5^{\circ} \mathrm{Celsius}$ des eaux de surface dans la mer des Caraïbes, le golfe du Mexique et dans l'Atlantique nord, augmente la fréquence des cyclones tropicaux de $40 \%$. D'autres travaux établissent le lien causal probable avec le changement du climat d'origine anthropique ; l'impact du $\mathrm{CO}_{2}$ et du réchauffement influent sur les précipitations et l'intensité des ouragans (Knuston et Tuleya, 2004) par exemple, ou encore les réanalyses de Kossin et al. (2007) sur les records historiques de l'activité cyclonique. De plus, pour Elsner et al. (2008) une augmentation de $1^{\circ} \mathrm{C}$ de la température de surface océanique entraîne une augmentation de la fréquence globale des cyclones forts (avec des vents supérieurs 51 $\mathrm{m} / \mathrm{s}$, i.e. $184 \mathrm{~km} / \mathrm{h}$ ), qui passent de 13 à 17 par an, soit une hausse de $31 \%$, inférieure à celle prévue par Emanuel en 2005. Ce constat est statistiquement vérifié pour les cyclones les plus violents, dont l'intensité s'accentue, en particulier dans l'Atlantique nord.

Cependant, il existe des incertitudes sur la capacité à discerner les tendances à long terme de l'activité cyclonique tropicale (Mann et al., 2007). Si le réchauffement des mers suggère que l'océan a plus d'énergie à convertir en vent de cyclone tropical, l'intensité 
des cyclones n'est pas due uniquement aux températures océaniques; la compréhension des raisons pour lesquelles certains cyclones se renforcent parfois très rapidement, alors que d'autres ne le font pas reste partielle (Elsner et al., 2008).

31 Quels que soient les causes de l'augmentation de l'intensité des cyclones, les risques eux, sont spatialement et temporellement différents.

\section{Le risque cyclonique lié à Katrina (2005) et Gustav et Ike (2008)}

\subsection{Les aléas Katrina, Gustav et Ike sont assez comparables}

Les ouragans Gustav et Ike sont comparables à l'ouragan Katrina dans la mesure où ils ont occasionné de nombreux dégâts et endeuillé les îles antillaises, avant d'aborder les côtes américaines du Golfe du Mexique. Leurs trajectoires sont légèrement différentes (fig. 4 \& 5), mais tous trois finissent leur parcours maritime dans le golfe du Mexique, alors que d'autres cyclones longent la côte Atlantique des Etats-Unis comme Hanna (28/08 au 7/09/2008) ou comme Hugo en 1989 (voir l'année: http:// www.wunderground.com/hurricane/at1989.asp).

Ike est l'événement qui a duré le plus longtemps, 14 jours contre 9 pour Katrina et Gustav, mais Katrina a été l'ouragan dont la pression a été minimale et les vents les plus forts (Fig. 6). Katrina, Gustav et Ike ont tous les trois atteint leur intensité maximale entre le $6^{\mathrm{e}}$ et le $7^{\mathrm{e}}$ jour. La corrélation linéaire entre les vents d'une part, et les pressions d'autre part, de Katrina et des deux cyclones est bonne et supérieure à 0,61. En revanche Ike et Gustav sont mal corrélés entre eux. 
Figure 6 - Analyse comparée de la pression minimale enregistrée (en haut) et de l'intensité des vents (en bas) entre les ouragans Katrina (2005), Gustav et lke (2008).

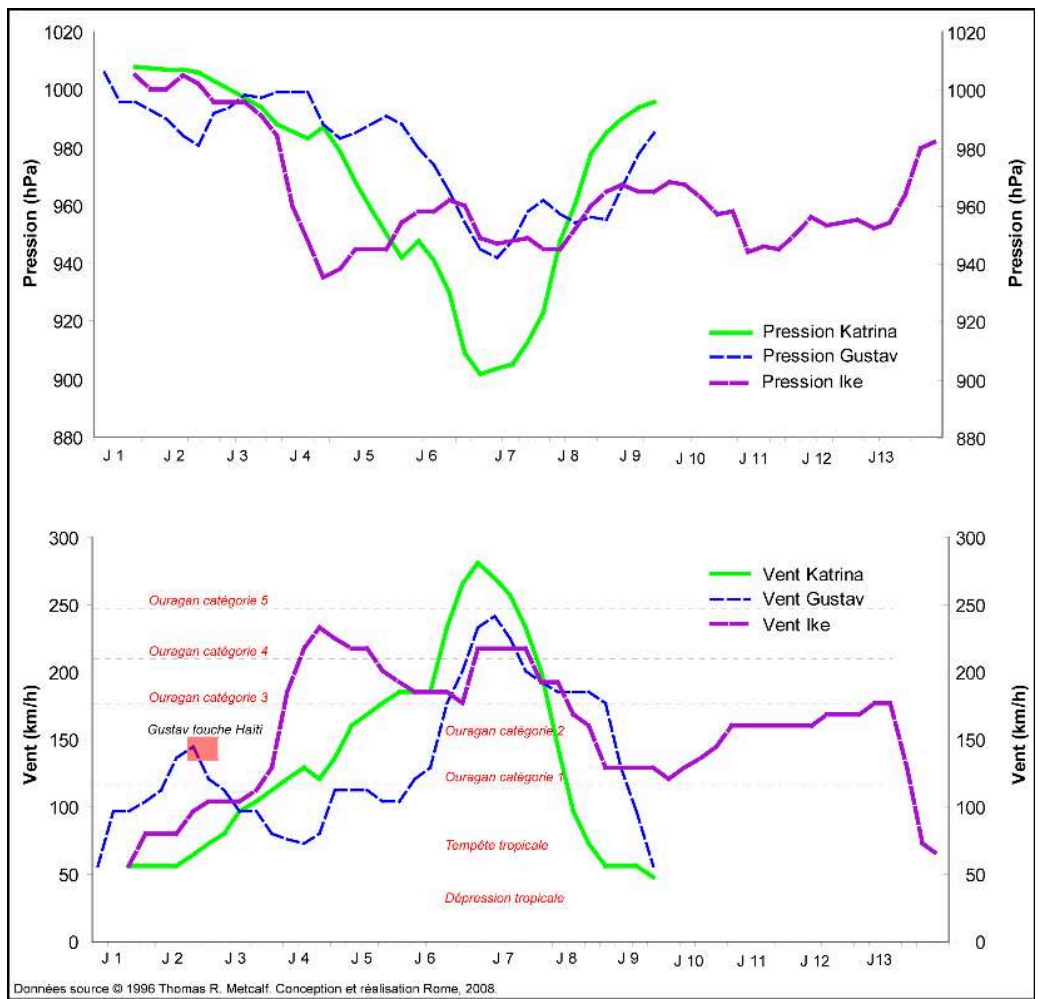

Au-delà de l'aléa, c'est surtout la vulnérabilité qui est très différente.

\subsection{Une vulnérabilité spatiale et temporelle variable}

La vulnérabilité associée aux cyclones tropicaux est exprimée ici restrictivement par le nombre de décès (tab. 2). Au moment de la rédaction de cet article, l'ensemble des dégâts occasionnés est encore difficile à chiffrer. 
Tableau 2 - Evaluation partielle de l'aléa et de la vulnérabilité aux cyclones Katrina, Gustav et lke

\begin{tabular}{|c|c|c|c|}
\hline & Katrina & Gustav & Ike \\
\hline $\begin{array}{l}\text { Date, durée et lieu } \\
\text { de formation }\end{array}$ & $\begin{array}{l}23-31 \text { août } 2005 \\
\text { Bahamas; } \\
23.1 \mathrm{~N}-31.1 \mathrm{~W}\end{array}$ & $\begin{array}{l}25 \text { août }-02 \text { septembre } \\
2008 \\
16.3 \mathrm{~N}-71.0 \mathrm{~W} \\
\end{array}$ & $\begin{array}{l}01-14 \text { septembre } 2008 \text {; } \\
17.60 \mathrm{~N}-39.50 \mathrm{~W}\end{array}$ \\
\hline Trajectoire & $\begin{array}{l}\text { Nord des Grandes Antilles, } \\
\text { Floride et Golfe du Mexique }\end{array}$ & $\begin{array}{l}\text { Sud des Grandes Antilles } \\
\text { et Golfe du Mexique }\end{array}$ & $\begin{array}{l}\text { Nord des Petites Antilles, } \\
\text { Cuba, et Golfe du Mexique }\end{array}$ \\
\hline $\begin{array}{l}\text { Pression minimale } \\
\text { enregistrée et date }\end{array}$ & $\begin{array}{l}945 \mathrm{hPa} \text { le 26/08/2005 à } 9 \mathrm{~h} \\
\text { GMT }\end{array}$ & $\begin{array}{l}955 \mathrm{hPa} \text { le } 30 / 08 / 2008 \text { à } \\
12 \mathrm{~h} \mathrm{GMT}\end{array}$ & $\begin{array}{l}935 \mathrm{hPa} \text { le 04/09/2008 à } 9 \mathrm{~h} \\
\text { GMT }\end{array}$ \\
\hline $\begin{array}{l}\text { Vitesse maximale } \\
\text { du vent (en } \\
\text { arrivant sur la } \\
\text { terre ferme) }\end{array}$ & $\begin{array}{l}240 \mathrm{~km} / \mathrm{h} \text { en arrivant sur } \\
\text { les côtes de Louisiane, } \\
280 \mathrm{Km} / \mathrm{h} \text { quelques heures } \\
\text { plus tôt }\end{array}$ & $\begin{array}{l}225 \mathrm{~km} / \mathrm{h} \text { le } 30 / 08 / 2008 \text { sur } \\
\text { Cuba }\end{array}$ & $\begin{array}{l}193 \mathrm{~km} / \mathrm{h} \text { le } 08 / 09 / 2008 \text { à } \\
\text { l'approche de Cuba }\end{array}$ \\
\hline $\begin{array}{l}\text { Type de cyclone et } \\
\text { stade }\end{array}$ & $\begin{array}{l}\text { Ouragan de catégorie } 5, \\
\text { rétrogradé en catégorie } 4 \\
\text { en arrivant en Louisiane }\end{array}$ & $\begin{array}{l}\text { Tempête tropicale puis } \\
\text { ouragan de catégorie } 4\end{array}$ & Ouragan de catégorie 3 \\
\hline $\begin{array}{l}\text { Diamètre du } \\
\text { cyclone }\end{array}$ & plus de $650 \mathrm{~km}$ & $370 \mathrm{~km}$ & $800 \mathrm{~km}$ \\
\hline Victimes & $\begin{array}{l}1833 \text { morts et } 705 \\
\text { disparus aux USA; } 81000 \\
\text { millions de \$ }\end{array}$ & $\begin{array}{l}8 \text { morts en République } \\
\text { Dominicaine, } 66 \text { à Haîti, } 11 \\
\text { en Jamaïque et } 7 \text { en } \\
\text { Louisiane : } 92 \text { au total }\end{array}$ & $\begin{array}{l}66 \text { tués à Haïti, } 4 \text { à Cuba, } \\
52 \text { aux Etats }{ }^{-} \text {nis, soit } 122 \\
\text { au total }\end{array}$ \\
\hline $\begin{array}{l}\text { Mesures d'alerte et } \\
\text { plans d'évacuation }\end{array}$ & $\begin{array}{l}\text { Oui aux USA, mais } \\
\text { partielles }\end{array}$ & Oui à Cuba, peu à Haiti & Oui à Cuba, peu à Haiti \\
\hline
\end{tabular}

Par ailleurs, les informations sur les mesures de prévention et d'évacuation sont partielles.

\subsection{Un risque élevé fonction de la pauvreté ou du manque de respect des consignes de sécurité ?}

37 Le risque cyclonique dépend certes de l'intensité de l'aléa, mais aussi des mesures de prévention, d'évacuation préalable, voire la capacité que présente la société affectée à surmonter le cyclone.

L'ouragan de catégorie 2 Dean, n'a fait aucune victime lors de son passage sur le département français d'outre mer martiniquais en août 2007, mais il a dévasté les bananeraies et fortement perturbé l'économie locale. Or aux Antilles françaises, les nouvelles consignes de sécurité et plan d'alertes cycloniques établies en 1999 par les services préfectoraux sont très efficaces; il existe une véritable «culture du risque cyclonique » et chacun connaît les gestes à faire avant, pendant et après le passage d'un ouragan.

39 Quelques centaines de kilomètres au nord-ouest, la république de Haïti, l'un des états les plus pauvres de la planète, ne bénéficie pas d'un tel niveau d'alerte, ne serait-ce parce les informations préventives, lorsqu'elles parviennent à la population, ne peuvent pas être correctement véhiculées auprès de tous (problème de transistor, de piles, de réflexes acquis de survie).

40 Cuba n'a eu à déplorer "que » sept décès lors des deux ouragans Gustav et Ike, grâce aux évacuations préventives massives des littoraux effectuées par les autorités qui ont permis d'épargner la population. Sept morts c'est toujours trop, mais «peu » comparativement à Haïti qui perd près de 570 âmes durant la saison cyclonique 2008 ! Plus de 2,5 millions de cubains ont été mis à l'abri grâce à plus de 10000 véhicules utilisés à cet effet. Au total, 87000 personnes ont été mobilisées pour la sauvegarde des vies humaines. 
41 Aux États-Unis, État tout puissant sur la scène mondiale, la majeure partie des victimes d'ouragan est due aux promeneurs curieux qui bravant les mesures de confinement. Dans le cas des victimes liées à la rupture de la digue du lac Pontchartrain à la Nouvelle Orléans, le problème est autre ; mais les procédures d'évacuation avaient épargné les couches sociales les plus aisées qui ont pu se déplacer.

Un fort risque cyclonique semble donc, au moins en partie, lié à une forte vulnérabilité sociale plutôt qu'à un aléa phénoménal.

Dans l'océan Indien, cette vulnérabilité sociale aux typhons est également constatée. Durand-Dastès (2008) cite les actions et réactions des institutions humaines, c'est-à-dire les «forces» qu'elles peuvent mettre en œuvre, autant que l'ampleur des forces naturelles à l'origine de la catastrophe, comme composante pouvant jouer un rôle tout à fait notable dans le développement des conséquences économiques et sociales des aléas cycloniques. Trois domaines, assez différents par leurs dimensions spatiales et temporelles sont évoqués: la prévision, l'intervention des mesures à long terme capables de modifier les effets d'une catastrophe éventuelle et enfin, l'attitude gouvernementale.

\section{Conclusion}

La vulnérabilité aux cyclones tropicaux semble autant liée au manque ou à l'insuffisance de mesures de prévention et d'évacuation des populations des littoraux qu'à l'ampleur de l'aléa potentiellement catastrophique. L'ouragan Gustav est présenté comme l'un des plus violent depuis Katrina : or, grâce aux moyens mis en œuvre par les autorités américaines, ce cyclone de catégorie 2 lors de son arrivée sur les côtes de Louisiane, fait relativement peu de victimes.

Un fort risque cyclonique s'avèrerait alors surtout dépendant de l'importance de la vulnérabilité, elle-même liée à la capabilité des institutions à faire face à l'aléa.

\section{BIBLIOGRAPHIE}

Elsner J. B., Kossin J. P. \& Jagger T. H., 2008. The increasing intensity of the strongest tropical cyclones. Nature 455, 92-95.

Emanuel K. A., 2005. Increasing destructiveness of tropical cyclones over the past 30 years. Nature 436, 686-688.

Durand-Dastès F., 2008. Une catastrophe dans son contexte : l'ouragan Nargis dans la Baie du Bengale (avril-mai 2008). Cybergeo, Les échelles des catastrophes. URL : http://www.cybergeo.eu/ index20143.html.

Holland G. J. and Webster P. J., 2007. Heightened tropical cyclone activity in the North Atlantic: natural variability or climate trend? Phil. Trans. R. Soc. A. doi:10.1098/rsta.2007.2083. http:// webster.eas.gatech.edu/static/Papers/Webster2007a.pdf 
Knutson T. R. and Tuleya R. E., 2004. Impact of CO2-Induced Warming on Simulated Hurricane Intensity and Precipitation: Sensitivity to the Choice of Climate Model and Convective Parameterization. Journal of Climate, 17, $n^{\circ} 18: 3477-3495.15$ September 2004.

Kossin, J. P., Knapp K. R., Vimont D. J., Murnane R. J., and Harper B. A., 2007: A globally consistent reanalysis of hurricane variability and trends. Geophys. Res. Lett., 34, L04815, doi:

$10.1029 / 2006$ GL028836.

Mann M. E., Emanuel K.A.,. Holland G. J, and Webster P. J. Atlantic Tropical Cyclones Revisited. Eos, vol. 88, n 36, 4 September 2007 : 349-350. http://webster.eas.gatech.edu/static/Papers/ Webster2007b.pdf

Rome S., 2006. Aléa cyclonique et vulnérabilité des sociétés. Journées de Climatologie de la Commission « Climat et Société » du Comité National Français de Géographie, Nice, mars 2006.

Saunders M. A \& Lea A. S, 2008. Large contribution of sea surface warming to recent increase in Atlantic hurricane activity. Nature451, 557-560.

Webster P. J., Holland, G. J., Curry, J. A. \& Chang H.-R., 2005. Changes in Tropical Cyclone Number, Duration, and Intensity in a Warming Environment. Science, 309, 1844-1846, 16 September 2005.

Météofrance, 2008. Saison cyclonique 2008 sur l'Atlantique et en Caraïbe. http://www.meteo.fr/ temps/domtom/antilles/pack-public/cyclone/res_sais_cyc_2008.htm

AFP, 2008. Ouragan Ike : dégâts considérables au Texas mais le pire a été évité.14/09/2008.

\section{RÉSUMÉS}

Le cyclone Gustav qui aborde les côtes ouest de la Louisiane fin août 2008 est présenté comme l'un des plus intenses depuis Katrina qui a considérablement affecté la ville de la Nouvelle Orléans trois ans plus tôt. Or, si l'aléa cyclonique est comparable, la vulnérabilité l'est moins, notamment parce que la prévention est plus efficace. Cet article compare différents cyclones de l'Atlantique, en particulier Katrina, Gustav, Hanna et Ike, en différenciant l'aléa de la vulnérabilité, spécialement à Haïti et aux Etats-Unis.

Hurricane Gustav which approaches the Western coast of Louisiana at the end of August 2008 is presented like one of most intense since Katrina, which earlier has considerably affected the city of News Orleans three years ago. However, if the hazard is comparable, the vulnerability is less in particular because of an effective prevention. This article compares various hurricanes of the Atlantic Ocean, Katrina, Gustav, Hanna and Ike, by differentiating the natural hazard from the vulnerability, especially in Haiti and the United States.

\section{INDEX}

Mots-clés : Atlantique, cyclone, Ike, Katrina, ouragan

Keywords : Atlantic Ocean, cyclone, hurricane, Ike, Katrina 


\section{AUTEUR}

\section{SANDRA ROME}

Sandra Rome (sandra.rome@ujf-grenoble.fr) est géographe climatologue, et enseignantchercheur à l'Université Joseph Fourier Grenoble 1et est membre de l'UMR CNRS 5194 PACTE. Elle a publié récemment :

- ROME S., BIGOT S., CHAFFARD V. et BIRON P.-E., 2008 : Relation entre les températures de l'air et les températures du sol : l'exemple des Hauts Plateaux du Vercors. Actes du colloque de l'Association Internationale de Climatologie, 21. Montpellier, septembre 2008.

- BIGOT S. et ROME S., 2008 : Elaboration d'un atlas de l'enneigement à l'échelle du Vercors grâce à l'imagerie SPOT-VEGETATION. Actes du colloque de l'Association Internationale de Climatologie, 21. Montpellier, septembre 2008.

- Rome S. et Giorgetti J.-P., 2007 : La montagne corse et ses caractéristiques climatiques. La Météorologie $8^{\mathrm{e}}$ série, $\mathrm{n}^{\circ} 59$ - novembre 2007, 39-50.

- ROME S., 2006 : Aléa cyclonique et vulnérabilité des sociétés. Journées de Climatologie de la Commission « Climat et Société », Comité National Français de Géographie, Nice, mars 2006. 